\title{
Novel Approach for 3d Chemical and Elastic State Assessment of Alloyed Strained Nanostructures
}

\author{
A. Isaac ${ }^{*}$, L.A. Montoro ${ }^{*}$, A. Malachias ${ }^{*}$, G. Medeiros-Ribeiro ${ }^{* *}$, A.J. Ramirez* \\ *Bazilian Synchrotron Light Laboratory, C. P. 6192, Campinas, SP 13083-970, Brazil \\ ${ }^{* *}$ Hewlett-Packard Laboratories, Palo Alto, 1501 Page Mill Road CA 94304
}

Applications of nanostructured semiconductors in electronic and optoelectronic require a precise knowledge of the chemical composition and elastic state. Several growth methods and parameters leading to distinct kinetic conditions will produce different chemical and stress/strain distributions, which call for tools that can provide a complete, unambiguous, and high-resolution description of such nano-structures. These issues have been addressed by several authors using different techniques such as atomic force microscopy (AFM) combined with selective chemical etching, Xray absorption fine structure, TEM, and grazing incidence anomalous X-ray diffraction (GIXRD). However, still limited information is available on the 3D chemical composition and elastic state of such nanostructures. Energy-dispersive X-ray spectroscopy (XEDS) and electron energy loss spectroscopy (EELS) associated to STEM has been used to provide chemical information of individual islands. However, the reported results obtained using these techniques have limited spatial resolution and/or fail to provide information regarding the $3 \mathrm{D}$ composition distribution of the entire island.

Recently, we combined geometrical phase analysis (GPA) and focal series aberration corrected images obtained by HRTEM on $\mathrm{Ge}_{x} \mathrm{Si}_{1-\mathrm{x}} / \mathrm{Si}(001)$ islands, allowing the investigation of the twodimensional projected distortion maps and, from them, their respective lattice parameter components (parallel, $a_{\|}$and perpendicular, $a_{\perp}$ ) and projected chemical compositions, from two different crystallographic orientations [1,2]. Based on this approach, the 3D chemical distribution was elucidated for these nanostructures. Here is now reported a novel method that combines the chemical distribution projections obtained from HRTEM (Fig.1-a, c) to finite elements (FE) simulations to solve the 3D strain/stress state of the strained alloyed nanostructure (Fig. 2). The 3D chemical distribution was inferred in an iterative process, achieving sufficient agreement with sideview projections obtained by HRTEM (Fig. 1-b, d). Therefore, the combined use of HRTEM and FEM calculations overcomes the lack of experimentally accessible information and it opens new opportunities for the complete 3D understanding of strained alloyed nanostructures. This methodology can be applied for a large variety of strained crystalline systems, such as nanowires, epitaxial islands, quantum dots and wells, and partially relaxed heterostructures.

\section{References}

[1] L.A. Montoro et al., J. Phys Chem. C 113 (2009) 9018.

[2] L.A. Montoro et al., J. Phys Chem. C 114 (2010) 12409. 


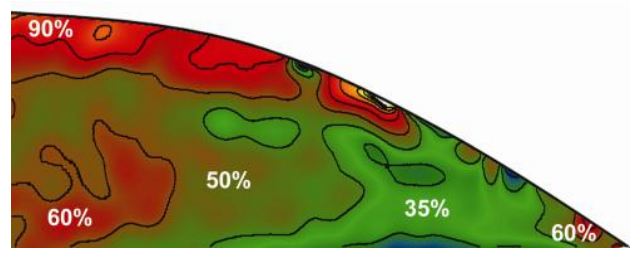

(a) [110]-direction, HRTEM

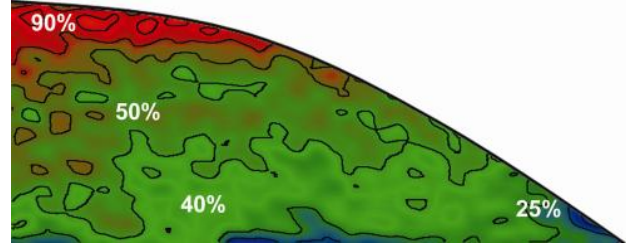

(c) [100]-direction, HRTEM

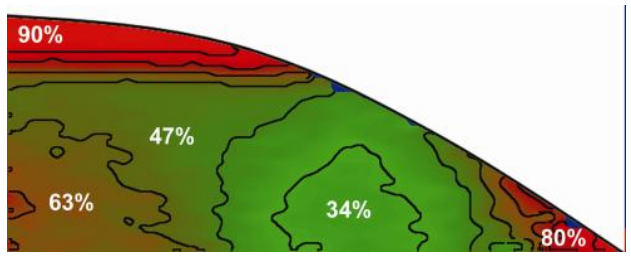

(b) [110]-direction, FEM

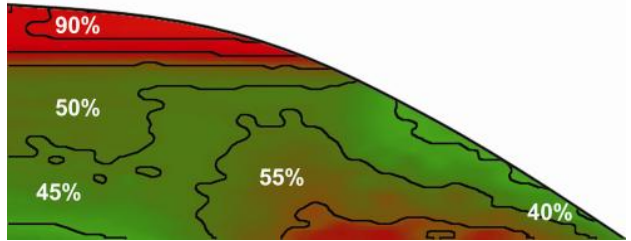

(d) [100]-direction, FEM

Fig. 1. Two-dimensional Ge-content $\left(\%_{\text {atom }}\right)$ maps at the projected directions of alloyed domeshaped islands.

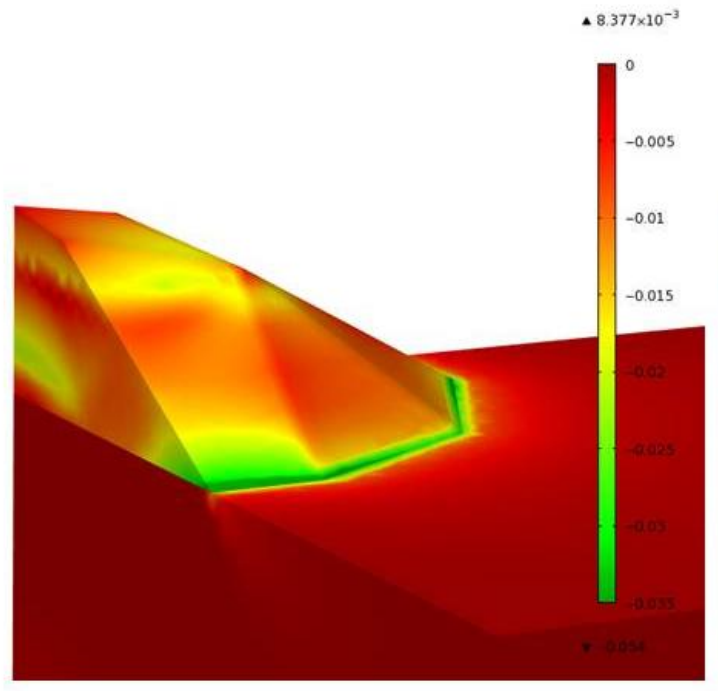

(a)

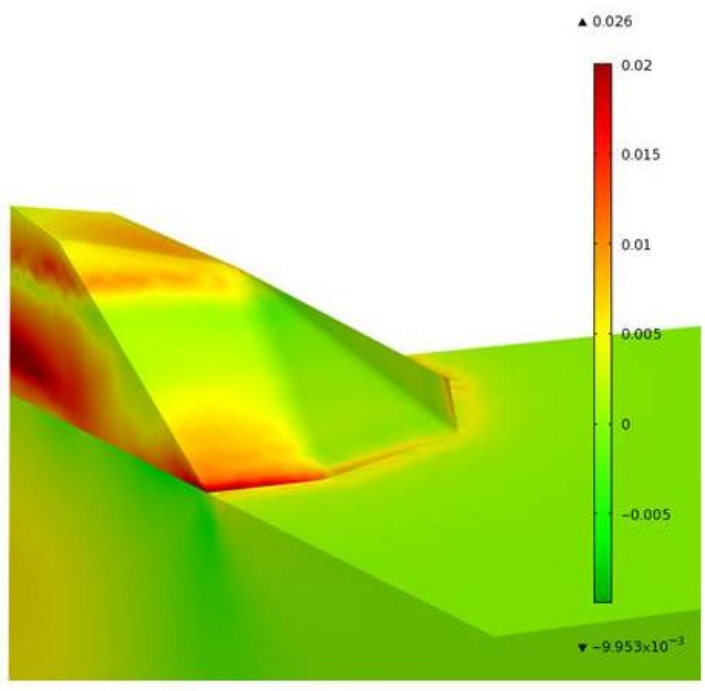

(b)

Fig. 2. Three-dimensional strain maps: (a) parallel and (b) perpendicular strain components. 